\title{
Efficacy of Magnesium Trihydrate of Ursodeoxycholic Acid and Chenodeoxycholic Acid for Gallstone Dissolution: A Prospective Multicenter Trial
}

Jong Jin Hyun*, Hong Sik Lee*, Chang Duck Kim*, Seok Ho Dong ${ }^{\dagger}$, Seung-OK Lee ${ }^{\ddagger}$, Ji Kon Ryu ${ }^{\S}$, Don Haeng Lee", Seok Jeong", Tae Nyeun Kim", Jin Lee ${ }^{\#}$, Dong Hee Koh", Eun Taek Park**, Inseok Lee ${ }^{\text {t† }}$, Byung Moo Yoo ${ }^{\ddagger \neq}$, and Jin Hong Kim ${ }^{\ddagger \neq}$

*Department of Internal Medicine, Korea University College of Medicine, ${ }^{\dagger}$ Department of Internal Medicine, Kyung Hee University School of Medicine, Seoul, ${ }^{\ddagger}$ Department of Internal Medicine, Chonbuk National University Medical School, Jeonju, ${ }^{s}$ Department of Internal Medicine, Seoul National University College of Medicine, Seoul, "Department of Internal Medicine, Inha University School of Medicine, Incheon,

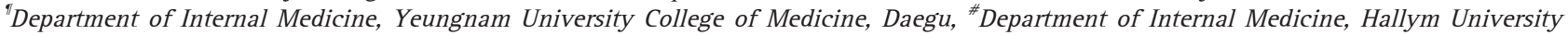
College of Medicine, Chuncheon, **Department of Internal Medicine, Kosin University College of Medicine, Busan, ${ }^{+t}$ Department of Internal Medicine, The Catholic University of Korea College of Medicine, Seoul, and ${ }^{\ddagger \pm}$ Department of Internal Medicine, Ajou University School of Medicine, Suwon, Korea

Background/Aims: Cholecystectomy is necessary for the treatment of symptomatic or complicated gallbladder (GB) stones, but oral litholysis with bile acids is an attractive alternative therapeutic option for asymptomatic or mildly symptomatic patients. This study was conducted to evaluate the efficacy of magnesium trihydrate of ursodeoxycholic acid (UDCA) and chenodeoxycholic acid (CDCA) on gallstone dissolution and to investigate improvements in gallstone-related symptoms. Methods: A prospective, multicenter, phase 4 clinical study to determine the efficacy of orally administered magnesium trihydrate of UDCA and CDCA was performed from January 2011 to June 2013. The inclusion criteria were GB stone diameter $\leq 15 \mathrm{~mm}$, GB ejection fraction $\geq 50 \%$, radiolucency on plain X-ray, and asymptomatic/mildly symptomatic patients. The patients were prescribed one capsule of magnesium trihydrate of UDCA and CDCA at breakfast and two capsules at bedtime for 6 months. The dissolution rate, response rate, and change in symptom score were evaluated. Results: A total of 237 subjects were enrolled, and 195 subjects completed the treatment. The dissolution rate was $45.1 \%$ and the response rate was $47.2 \%(92 / 195)$ after 6 months of administration of magnesium trihydrate of UDCA and CDCA. Only the stone diameter was significantly associated with the response rate. Both the symptom score and the number of patients with symptoms significantly decreased regardless of stone dissolution. Adverse events necessitating discontinuation of the drug, surgery, or endoscopic manage- ment occurred in 2.5\% (6/237) of patients. Conclusions: Magnesium trihydrate of UDCA and CDCA is a well-tolerated bile acid that showed similar efficacy for gallstone dissolution and improvement of gallstone-related symptoms as that shown in previous studies. (Gut Liver 2015;9:547-555)

Key Words: Gallstones; Dissolution; Ursodeoxycholic acid; Chenodeoxycholic acid

\section{INTRODUCTION}

Gallstone disease is still the most prevalent medical issue in the pancreatobiliary system. ${ }^{1,2}$ With the increase in the use of ultrasonography for screening purposes and evaluation of abdominal pain, gallstones are more frequently being detected. According to a study that looked into the demographic features of gallstone disease in Korea over the past 30 years, the total number of surgically treated gallstone patients rose three-folds during the study period. ${ }^{1}$ This increase was mostly attributable to the increase in the number of patients with gallbladder (GB) stones, which in turn can largely be ascribed to westernization of lifestyle and increase in body mass index (BMI), especially among the young patients. ${ }^{1,3}$ In fact, a study by Kim et al. ${ }^{4}$ showed that the prevalence of cholesterol gallstones in Korea proportionately increased as age decreased among those who underwent cholecystectomy $(35.6 \%$ in patients aged $\geq 50$ years vs $72.0 \%$ in patients aged $<50$ years).

\footnotetext{
Correspondence to: Hong Sik Lee

Division of Gastroenterology and Hepatology, Department of Internal Medicine, Korea University Anam Hospital, 73 Inchon-ro, Seongbuk-gu, Seoul 136-705, Korea

Tel: +82-2-920-5312, Fax: +82-2-953-1943, E-mail: hslee60@korea.ac.kr Received on January 9, 2015. Revised on February 25, 2015. Accepted on March 10, 2015. pISSN 1976-2283 eISSN 2005-1212 http://dx.doi.org/10.5009/gnl15015

(a) This is an Open Access article distributed under the terms of the Creative Commons Attribution Non-Commercial License (http://creativecommons.org/licenses/by-nc/4.0) which permits unrestricted non-commercial use, distribution, and reproduction in any medium, provided the original work is properly cited.
} 
Treatment of gallstone differs depending on the presence of symptoms, type of gallstone, and its location. Although laparoscopic cholecystectomy is the treatment of choice for symptomatic or complicated GB stones, oral litholysis with bile acids is an attractive alternative therapeutic option for asymptomatic or mildly symptomatic subgroups. ${ }^{2}$ Currently, the most widely used agents for oral litholysis in Korea are ursodeoxycholic acid (UDCA) alone or in combination with chenodeoxycholic acid (CDCA). The standard dose of UDCA prescribed for gallstone dissolution is 8 to $12 \mathrm{mg} / \mathrm{kg} /$ day. ${ }^{5}$ Dissolution therapy is recommended to be stopped after 6 months if the gallstones do not show any response, i.e., decrease in size. Dissolution therapy can be continued if gallstones are partially dissolved after 6 months, but should be stopped if complete dissolution is not achieved by 2 years. Complete dissolution rate varies among studies which ranges from $20 \%$ to $70 \%{ }^{6}$ A meta-analysis on dissolution therapy showed that complete dissolution occurred in 38\% of patients when UDCA was given in doses greater than $7 \mathrm{mg} / \mathrm{kg} /$ day for more than 6 months. ${ }^{7}$ UDCA is generally well tolerated and does not cause any significant adverse effects although there have been reports on the acquisition of gallstone calcification during therapy. ${ }^{8-10}$ As for the combination therapy with UDCA and CDCA, the dose used for gallstone dissolution is $5 \mathrm{mg} / \mathrm{kg} /$ day each or $6 \mathrm{mg} / \mathrm{kg} /$ day each. ${ }^{5,10,11}$ The recommended duration of therapy is same as that of UDCA alone. Previous studies have shown that complete dissolution occurred in about 30\% to 50\% after 2 years of therapy, ${ }^{5,10}$ and that the mean dissolution rate at 6 months and 12 months were 44\% to 82\% and 53\% to 100\%, respectively. ${ }^{5,11}$ Compared to UDCA alone, diarrhea occurred more frequently in those treated with combination of UDCA and CDCA. These aforementioned previous studies have been conducted with the Western population, ${ }^{5,10,11}$ but data on its efficacy in non-Western population are lacking. Therefore, this phase 4, prospective, open-label, noncomparative, multicenter study was conducted to evaluate the gallstone dissolution efficacy and improvement of gallstone-related symptoms in Korean population.

\section{MATERIALS AND METHODS}

\section{Patients}

A prospective, multicenter, phase 4 clinical study was performed from January 2011 to June 2013 at 10 tertiary referral centers in Korea: Korea University Anam Hospital, Kyung Hee University Hospital, Chonbuk National University Hospital, Seoul National University Hospital, Inha University Hospital, Yeungnam University Hospital, Hallym University Hospital, Kosin University Hospital, Seoul St. Mary's Hospital, and Ajou University Hospital. The inclusion criteria were (1) GB stones $\leq 15 \mathrm{~mm}$ in diameter detected on abdominal ultrasonography, (2) GB ejection fraction (EF) $\geq 50 \%$ on diisopropyl iminodiacetic acid scan, (3) no visible GB stone on plain abdominal Xray, and (4) asymptomatic or mild symptomatic patients (pain that is less than 5 on a visual analogue scale, occurs less than 3 times a week, and each lasting less than 30 minutes). The following patients were excluded from the study: age under 18 years, patients with moderate to severe symptoms, complicated GB stones, patients with severe comorbidity, morbidly obese patients (BMI $\geq 35 \mathrm{~kg} / \mathrm{m}^{2}$ ), patents with history of having undergone gastrointestinal operation, patients with jaundice, and abnormal liver function test (aminotransferase $>2$ times the upper limit of normal).

\section{Study drug and study design}

This study was an open label study that looked into the efficacy and safety of orally administrated magnesium trihydrate of CDCA and UDCA (CNU ${ }^{\circledast}$; Myungmoon Pharmaceutical Company, Seoul, Korea). Each capsule of $\mathrm{CNU}^{\circledR}$ consists of 114 $\mathrm{mg}$ of UDCA and $114 \mathrm{mg}$ of CDCA. CNU ${ }^{\circledR}$ was prescribed to be taken one capsule at breakfast and two capsules at bedtime. The total duration of treatment was 6 months.

After enrollment, patients were asked to visit the outpatient department at 1, 3, and 6 months. During each visit, symptom score was measured and physical examination was performed. Patients were evaluated for the presence of nausea, indigestion, epigastric fullness, biliary pain, and fatigue. Symptom was also assessed at 2 weeks through telephone survey. Symptom score was measured using a visual analogue scale ranging from 0 to 10. Aspartate aminotransferase, alanine aminotransferase, alkaline phosphatase, $\gamma$-glutamyl transferase, and serum creatinine levels were checked during each visit. Follow-up abdominal ultrasonography was performed 6 months after completion of dissolution therapy and evaluated for GB stone number, largest diameter of GB stone, and presence of GB wall thickening and GB polyp. This study was approved by Institutional Review Board of each hospital.

\section{Assessment of efficacy and outcome definitions}

Abdominal ultrasonography was performed to assess GB stone size, and the radius ( $\mathrm{r}$ ) of the largest stone observed at baseline and at 6 months was used to calculate representative GB stone volume. GB stone volume was measured using the following equation as for a sphere: $4 / 3 \pi \times \mathrm{r}^{3}$. Dissolution rate was defined as percentage reduction in the GB stone volume. As for sludge, percentage reduction in number or area was used as an estimate to assess dissolution rate. Complete dissolution was defined as absence of GB stone on follow-up abdominal ultrasonography. Partial dissolution of GB stone was defined as reduction in stone volume of $>50 \%$. Response to therapy was defined as complete dissolution or partial dissolution.

To evaluate improvement in gallstone-related symptoms, changes in the symptom score and number of patients with symptoms were compared with the baseline data. The period during which greatest improvement occurred was also analyzed. Symptom improvement was analyzed according to the response to therapy. 


\section{Adverse event assessment}

The patients were informed of possible adverse events that could arise during $\mathrm{CNU}^{\circledR}$ treatment. Occurrence and severity of any adverse events were first evaluated at 2 weeks through telephone survey. If patients were intolerable to the prescribed medication or complained of noteworthy adverse events at any point during the course of medical litholysis, they were asked to withhold the medication and advised to return to the outpatients department. If a patient developed any gallstone-related complications, i.e., pancreatitis, cholangitis or cholecystitis, medication was stopped and relevant treatment was given: referral to surgeon for cholecystectomy or endoscopic retrograde cholangiopancreatography for stone removal.

\section{Statistical analysis}

Statistical analyses were performed using IBM SPSS Statistics version 20.0 (IBM, Armonk, NY, USA). Data are expressed as mean \pm SD or number (\%). Continuous and categorical variables were compared using the Mann-Whitney U test and chisquare test, respectively. Comparison on the number of patients with symptoms before and after the treatment was assessed by McNemar test. Comparison on the change of symptom score was assessed by paired t-test. A two-sided probability value of $p<0.05$ was considered statistically significant. For multivariate analysis, binary logistic regression analysis was performed using variables for which $\mathrm{p}<0.1$ in univariate analysis or those considered to be relevant.

\section{RESULTS}

\section{Study subjects and baseline characteristics}

A total of 237 subjects met the inclusion criteria and were enrolled in the study (Fig. 1). Among these subjects, 42 subjects

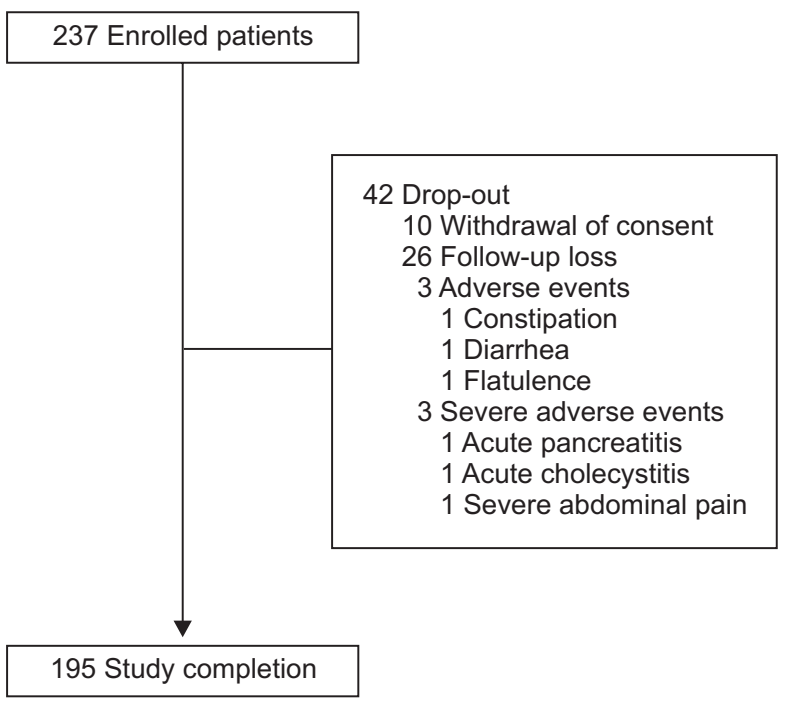

Fig. 1. Flow chart of the study population. could not complete the study due to following reasons: withdrawal of consent $(n=10)$, follow-up loss $(n=26)$, adverse events $(n=3)$, and severe adverse events $(n=3)$. Three patients stopped taking the medication due to adverse events which were constipation $(n=1)$, diarrhea $(n=1)$, and flatulence $(n=1)$. Medication was stopped in three subjects due to the occurrence of severe adverse events: acute pancreatitis $(n=1)$, acute cholecystitis $(n=1)$, and severe abdominal pain $(n=1)$. In the end, 195 subjects (86 male, 109 female) completed the study. Average age was $54.88 \pm 12.84$ years and their average BMI was $24.54 \pm 3.30$. Mild fatty liver was present in 56 subjects (28.7\%) and moderate fatty liver in 15 subjects (7.7\%). About half of the subjects had one or two stones and the remaining half had three or more stones. The average stone diameter was $9.11 \pm 3.76 \mathrm{~mm}$ with about half of the subjects having stones $\geq 10 \mathrm{~mm}$. The average GBEF was 73.19\% 0 13.57\% (Table 1).

Table 1. Baseline Characteristics of the Enrolled Patients

\begin{tabular}{|c|c|}
\hline Characteristic & Enrolled patients $(n=195)$ \\
\hline Female sex & 109 (55.9) \\
\hline Age, yr & $54.88 \pm 12.84$ \\
\hline Body weight, kg & $65.50 \pm 11.28$ \\
\hline BMI, $\mathrm{kg} / \mathrm{m}^{2}$ & $24.54 \pm 3.30$ \\
\hline Smoker & $29(14.9)$ \\
\hline Alcohol drinker & $50(25.6)$ \\
\hline Diabetes & $26(13.3)$ \\
\hline Hypertension & $53(27.2)$ \\
\hline \multicolumn{2}{|l|}{ Fatty liver } \\
\hline Normal & $124(63.6)$ \\
\hline Mild & $56(28.7)$ \\
\hline Moderate & $15(7.7)$ \\
\hline \multicolumn{2}{|l|}{ Stone no. } \\
\hline 1 & $79(40.5)$ \\
\hline 2 & $19(9.7)$ \\
\hline 3 & $23(11.8)$ \\
\hline$\geq 4$ & 74 (37.9) \\
\hline Stone diameter, mm & $9.11 \pm 3.76$ \\
\hline \multicolumn{2}{|c|}{ Stone size distribution, $\mathrm{mm}$} \\
\hline$<5$ & $21(10.8)$ \\
\hline$\geq 5$ to $<10$ & $79(40.5)$ \\
\hline$\geq 10$ & 95 (48.7) \\
\hline GBEF & $73.19 \pm 13.57$ \\
\hline AST, IU/L & $24.16 \pm 13.81$ \\
\hline ALT, IU/L & $24.18 \pm 23.72$ \\
\hline ALP, IU/L & $90.36 \pm 59.93$ \\
\hline GGT, IU/L & $40.76 \pm 52.35$ \\
\hline
\end{tabular}

Data are presented as number (\%) or mean \pm SD.

BMI, body mass index; GBEF, gallbladder ejection fraction; AST, aspartate aminotransferase; ALT, alanine aminotransferase; ALP, alkaline phosphatase; GGT, $\gamma$-glutamyl transpeptidase. 


\section{Response and dissolution rate of $\mathrm{CNU}^{\circledR}$}

Dissolution rate, measured as percentage reduction in volume, was $45.1 \%$. Complete dissolution was achieved in 12.8\% (25/195) and partial dissolution occurred in 34.4\% (67/195) after administration of $\mathrm{CNU}^{\circledR}$ for 6 months. Thus, the overall response rate was 47.2\% (92/195) (Table 2). When analyzed according to the size of the gallstone, complete dissolution occurred in were 47.6\% (10/21), 15.2\% (12/79), and 3.2\% (3/95) of patients with gallstones measuring $\leq 5 \mathrm{~mm}, 5-10 \mathrm{~mm}$, and $10-15 \mathrm{~mm}$, respectively. When only GB sludge was considered, dissolution rate was $57.1 \%$ and the overall response rate was $60.5 \%$ (26/43). Complete dissolution was achieved in 30.2\% (13/43) and partial dissolution occurred in another 30.2\% (13/43).

\section{Factors related to response to dissolution therapy}

Univariate analysis showed that neither BMI $(\mathrm{p}=0.847)$ nor

Table 2. Efficacy after $\mathrm{CNU}^{\circledR}$ Treatment

\begin{tabular}{lc}
\hline Response to dissolution therapy & No. (\%) \\
\hline Response & $92(47.2)$ \\
Complete dissolution & $25(12.8)$ \\
Partial dissolution & $67(34.4)$ \\
No response & $103(52.8)$ \\
\hline
\end{tabular}
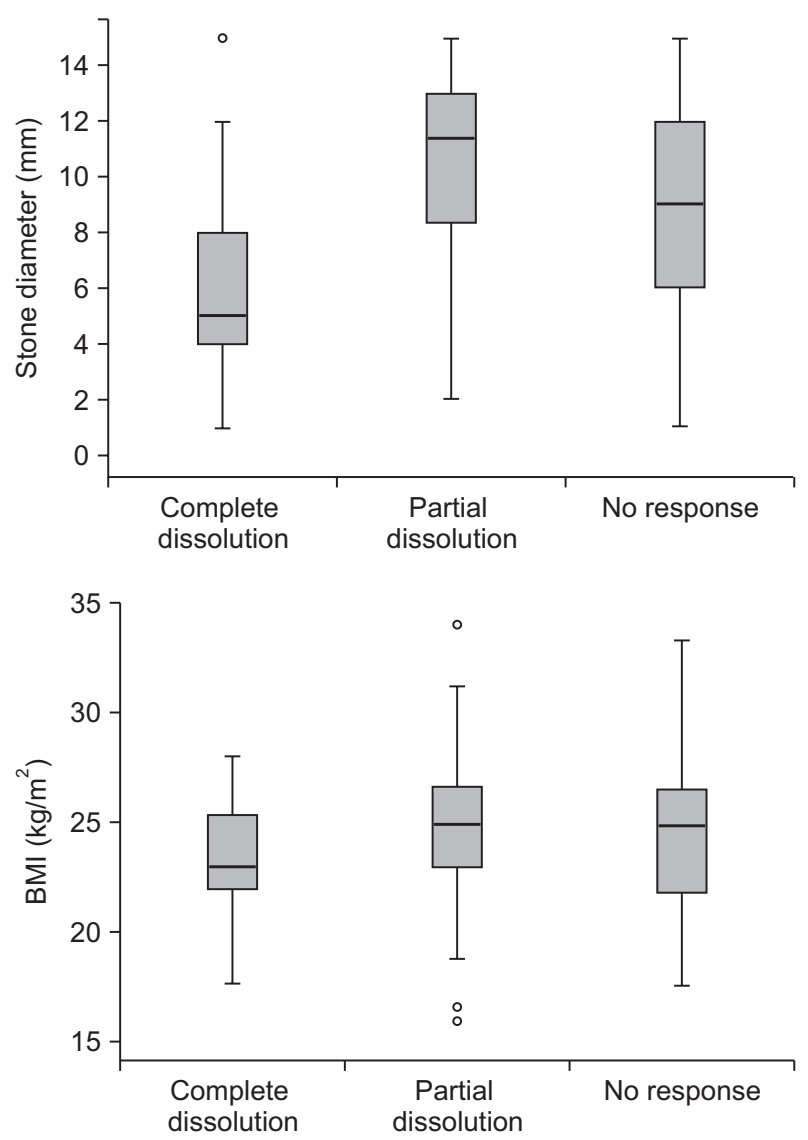

GBEF ( $\mathrm{p}=0.752$ ) was associated with response to dissolution therapy. Only stone diameter ( $\mathrm{p}=0.097$ ) showed some tendency with response to dissolution therapy (Fig. 2). On multivariate analysis, only stone diameter was shown to be significantly associated with response to dissolution therapy at 6 months after $\mathrm{CNU}^{\circledast}$ administration ( $\beta, 1.078$; odds ratio, 2.937; 95\% confidence interval, 1.067 to $8.085 ; \mathrm{p}=0.037$ ) (Table 3 ).

\section{Symptom improvement after $\mathrm{CNU}^{\circledR}$ administration}

The number of patients complaining of nausea, indigestion, epigastric fullness, biliary pain, and fatigue all significantly decreased after treatment with $\mathrm{CNU}^{\circledR}$ for 6 months (Fig. 3A). The decrease in the number of patients with symptoms was greatest during the first 2 weeks of therapy (Fig. 3B). Similar findings were observed with the symptom score. Symptom scores of nausea, indigestion, epigastric fullness, biliary pain, and fatigue all significantly improved after treatment with $\mathrm{CNU}^{\circledR}$ for 6 months (Fig. 3C). Symptom score also showed greatest improvement during the first 2 weeks (Fig. 3D). When symptom improvement was analyzed according to the response to therapy, $\mathrm{CNU}^{\circledR}$ treatment showed significant symptomatic improvement regardless stone dissolution; there were no difference in symptom improvement between response group and no response group regarding nausea $(\mathrm{p}=0.326)$, indigestion $(\mathrm{p}=0.352)$, fullness $(p=0.256)$, biliary pain $(p=0.152)$, and fatigue $(p=0.172)$.

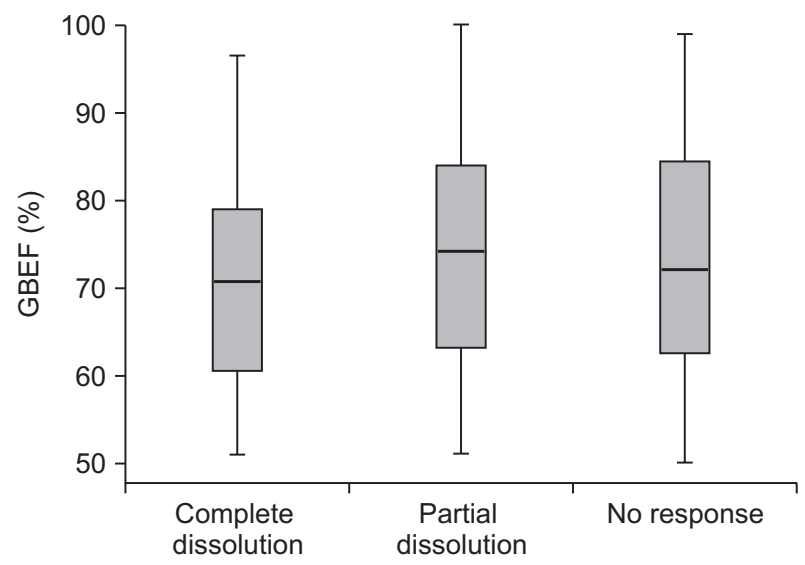

Fig. 2. Factors associated with the response to dissolution therapy. GBEF, gallbladder ejection fraction; BMI, body mass index. 
Table 3. Comparison between the Response Group and the No Response Group

\begin{tabular}{|c|c|c|c|}
\hline & $\begin{array}{l}\text { Response group } \\
\quad(\mathrm{n}=92)\end{array}$ & $\begin{array}{l}\text { No response group } \\
\qquad(\mathrm{n}=103)\end{array}$ & p-value \\
\hline Female sex & $50(54.3)$ & 59 (57.3) & 0.680 \\
\hline Age, yr & $55.84 \pm 13.17$ & $54.01 \pm 12.54$ & 0.320 \\
\hline BMI, $\mathrm{kg} / \mathrm{m}^{2}$ & $24.50 \pm 3.22$ & $24.59 \pm 3.40$ & 0.847 \\
\hline Fatty liver & & & 0.226 \\
\hline Normal & $64(69.6)$ & $60(58.3)$ & \\
\hline Mild & $23(25.0)$ & $33(32.0)$ & \\
\hline Moderate & $5(5.4)$ & $10(9.7)$ & \\
\hline GB wall thickening & $4(4.3)$ & $5(4.9)$ & 0.571 \\
\hline Stone no. & & & 0.659 \\
\hline 1 & $35(38.0)$ & $44(42.7)$ & \\
\hline 2 & $10(10.9)$ & $9(8.7)$ & \\
\hline 3 & $9(9.8)$ & $14(13.6)$ & \\
\hline$\geq 4$ & $38(41.3)$ & $36(35.0)$ & \\
\hline Stone diameter, mm & $9.24 \pm 3.93$ & $8.99 \pm 3.62$ & 0.650 \\
\hline Stone size distribution, $\mathrm{mm}$ & & & 0.097 \\
\hline$<5$ & $14(15.2)$ & $7(6.8)$ & \\
\hline$\geq 5$ to $<10$ & $32(34.8)$ & $47(45.6)$ & \\
\hline$\geq 10$ & $46(50.0)$ & 49 (47.6) & \\
\hline GBEF & $72.86 \pm 13.12$ & $73.48 \pm 14.01$ & 0.752 \\
\hline AST, IU/L & $23.26 \pm 7.78$ & $24.97 \pm 17.53$ & 0.389 \\
\hline ALT, IU/L & $22.21 \pm 13.58$ & $25.95 \pm 29.98$ & 0.272 \\
\hline ALP, IU/L & $88.28 \pm 61.87$ & $92.21 \pm 58.39$ & 0.649 \\
\hline GGT, IU/L & $46.47 \pm 57.46$ & $35.62 \pm 46.99$ & 0.159 \\
\hline
\end{tabular}

Data are presented as number (\%) or mean \pm SD.

BMI, body mass index; GB, gallbladder; GBEF, gallbladder ejection fraction; AST, aspartate aminotransferase; ALT, alanine aminotransferase; ALP, alkaline phosphatase; GGT, $\gamma$-glutamyl transpeptidase.

Although biliary pain significantly improved after 6 months in both groups (response vs no response, $p=0.008$ vs $p=0.021$ ), those with response showed much more improvement of biliary pain $(\mathrm{p}=0.017)$.

\section{DISCUSSION}

Dissolution therapy of GB stones has been performed since the 1970s with the introduction of CDCA and UDCA. ${ }^{12-14}$ CDCA was initially used more frequently, but because of dose-related diarrhea which was seen in approximately 50\% of patients, it is no longer used as monotherapy. In addition, treatment with CDCA also increased serum cholesterol and serum aminotransferase in a substantial proportion of patients. UDCA, on the other hand, causes significantly less diarrhea, does not increase serum cholesterol, and has no hepatotoxicity. ${ }^{15}$ Therefore, the use of CDCA alone has been abandoned and UDCA is currently the most widely used bile acid for gallstone dissolution. To increase dissolution efficacy and decrease the side effects of CDCA, CDCA and UDCA have been combined at a lower dose and this combination has been shown to be more efficacious or at least similar to monotherapy with UDCA., ${ }^{5,10,11}$ Therefore, the combination of CDCA and UDCA (CNU ${ }^{\circledR}$ ) was chosen to evaluate dissolution efficacy, and the result of our study performed with the Korean population shows that the mean dissolution rate was $45.1 \%$ and complete dissolution was achieved in $12.8 \%$ after 6 month of dissolution therapy with $\mathrm{CNU}^{\circledR}$. This is similar to the result by Petroni et al. ${ }^{5}$ in which the mean dissolution rate was 44\% and complete dissolution occurred in about $8 \%$ at 6 months after combination therapy with UDCA and CDCA. However, complete dissolution rate in our study was lower than that of the study by Podda et al. ${ }^{10}$ in which complete dissolution occurred in 52\% for smaller stones ( $\leq 5 \mathrm{~mm}$ ) and in $51 \%$ for larger stones ( 5 to $15 \mathrm{~mm}$ ) at 6 months. In addition, the experiment conducted by Jazrawi et al. ${ }^{11}$ also showed higher mean dissolution rate ( $82 \%$ vs $45.1 \%$ ) and the overall response (70\% vs $60.5 \%$ ) compared to our study at 6 months. It is well known that the prevalence of gallstone and gallstone composition shows geographical and ethnic variations. Whereas cholesterol stones constitute the majority of stones in the Western and de- 

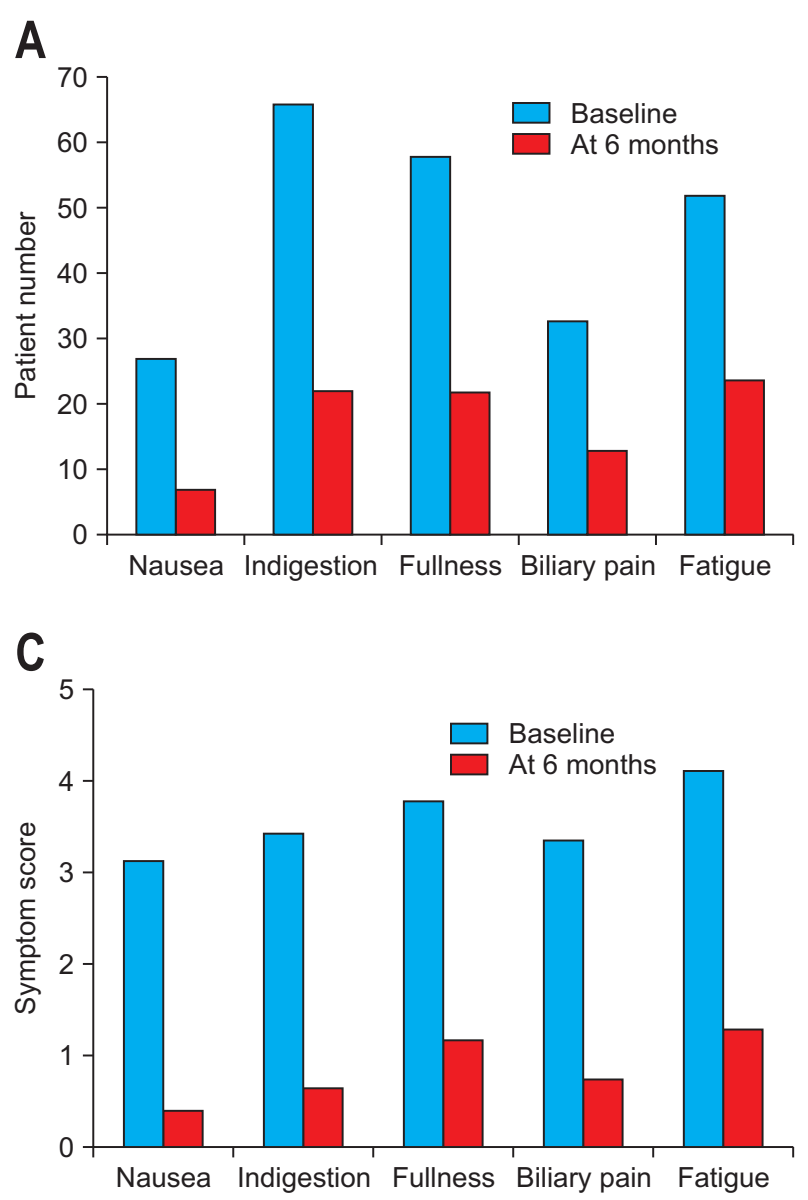
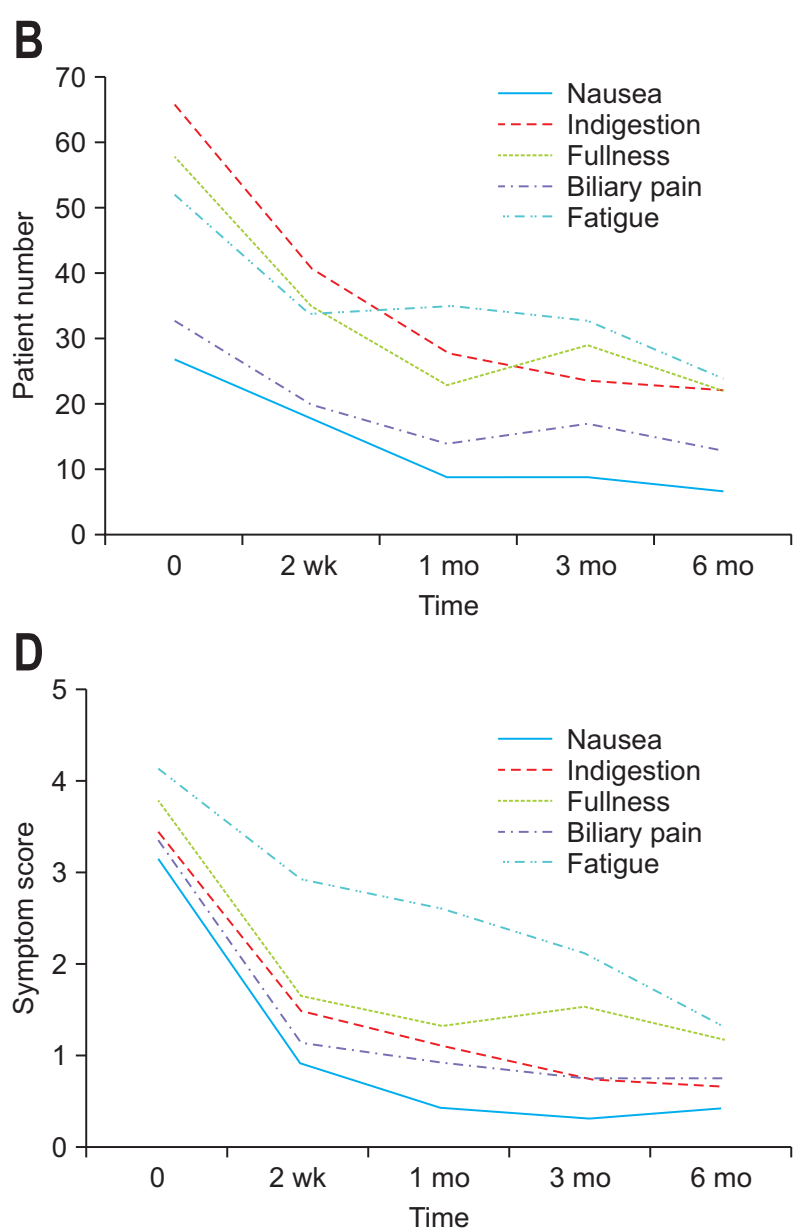

Fig. 3. Symptom improvement with $\mathrm{CNU}^{\circledR}$ treatment. Both the number of patients with symptoms (A) and the symptom score (C) significantly decreased at 6 months after treatment with $\mathrm{CNU}^{\circledR}$ compared with the baseline ( $\mathrm{p}<0.001$ for all symptoms). The decrease in the number of patients with symptoms showed the greatest decrease during the first 2 weeks (B). A similar trend was also observed with the symptom score (D).

veloped counties, ${ }^{16}$ pigment stones resistant to dissolution by bile acids are more predominant in the Eastern countries. ${ }^{4} \mathrm{Al}-$ though the prevalence of gallstone disease is becoming similar to that of the Western countries owing to the westernization of lifestyle and improvement in socioeconomic status, the relative proportion of patients with cholesterol gallstones is still lower than that of the West. ${ }^{4,17}$ Nevertheless, the dissolution rate and proportion of patients with complete dissolution at 6 months was comparable to the result by Petroni et al. ${ }^{5}$ which was performed with European population.

Although the proportion of patients with cholesterol gallstones has increased, about half of the patients did not respond to oral litholysis. Apart from the fact that the relative proportion of patients with cholesterol gallstones in Korea (49.0\% to $58.1 \%$ ) is still lower than that of the West, ${ }^{4,17}$ the reasons for ineffective litholysis could be due to the lack of discernibility of plain abdominal X-ray to rule out gallstone calcification. It is well known that only cholesterol gallstones are amenable to dissolution with orally administered bile acids. Prior to initiating dissolution therapy, plain abdominal X-ray is routinely carried out to select those with radiolucent stones as we have done in the present study. However, several studies have shown that up to $20 \%$ of pigment gallstones appear radiolucent on plain abdominal X-ray. ${ }^{18,19}$ Moreover, it has been demonstrated that no less than 50\% of gallstones which are radiolucent on plain abdominal X-ray appear to be hyperdense on abdominal computed tomography (CT) scan..$^{20,21}$ Many radiolucent cholesterol stones also have been shown to have high calcium content. ${ }^{22,23}$ Since high calcium content is one of the most important factors influencing dissolution efficacy, it would be recommendable to perform CT scan in order to discriminate cholesterol from pigment stone to some degree and to detect the composition of gallstone such as calcification.

In addition to the presence of calcium content that impedes gallstone dissolution, there are several important factors related to successful response to bile acid therapy. First is the size of the gallstone. Similar to previous studies, our study demonstrated that stone diameter was the only predictive parameter for gallstone dissolution. When analyzed according to the stone size, complete dissolution rates at 6 months for gallstones $\leq 5 \mathrm{~mm}$, 
5-10 mm, and 10-15 mm were 47.6\% (10/21), 15.2\% (12/79), and 3.2\% (3/95), respectively. Complete dissolution rate of $47.6 \%$ for smaller gallstones $(\leq 5 \mathrm{~mm})$ in the present study is similar to the previous studies by Petroni et al. ${ }^{5}$ and Podda et al. ${ }^{10}$ in which it was $47 \%$ and 52\%, respectively at 6 months. Second is the dose timing. In our study, the medication was prescribed to be taken one capsule of $\mathrm{CNU}^{\circledR}$ at breakfast and two capsules at bedtime. According to the study by Jazrawi et al., ${ }^{11}$ the most effective dose timing for dissolution therapy was to take the daily dose all together at bedtime than at mealtime in three divided dose. Dose timing adopted by Jazrawi et al. ${ }^{11}$ is based on the observation that since there is overnight reduction in hepatic bile acid secretion due to interruption in enterohepatic circulation, this leads to secretion of supersaturated hepatic bile. ${ }^{24-26}$ Thus, taking bile acids at bedtime would maintain hepatic bile acid secretion with resultant decrease in the secretion of supersaturated hepatic bile. In Korea, however, patients are used to taking the medication three times a day. Afternoon dose is often missed during working hours, especially when patients do not have symptoms that oblige them to take the medication. Thus, considering the situation in Korea, we have adopted the dose timing used by Podda et al., ${ }^{10}$ which increased compliance rate to more than $90 \%$. Third is the dose of bile acid. In the present study, all patients uniformly received three capsules of $\mathrm{CNU}^{\circledR}$ with each capsule containing $114 \mathrm{mg}$ of UDCA and $114 \mathrm{mg}$ of CDCA because this is the dose that is covered by the National Health Insurance Program of Korea. Since the dose used by previous studies for gallstone dissolution with the combination of CDCA plus UDCA was $5 \mathrm{mg} / \mathrm{kg} /$ day each or $6 \mathrm{mg} /$ $\mathrm{kg} /$ day each, patients weighing $57 \mathrm{~kg}$ to $68.4 \mathrm{~kg}$ would have received adequate dose if they were prescribed with three capsules of $\mathrm{CNU}^{\circledR}$. In our study, 83 patients (42.6\%) weighed more than $68.4 \mathrm{~kg}$ and they seem to have been underdosed. Since $\mathrm{CNU}^{\circledR}$ is a capsule that cannot be split or divided and comes only in one size, it might be preferable to develop the capsule into tablet or make additional capsules that contain different amounts of $\mathrm{CNU}^{\circledR}$. This would allow patients to receive a more effective dose and further enhance dissolution efficacy. Interestingly however, the dissolution rate $(45.8 \%)$ and response rate (47.0\%) in those weighing over $68.4 \mathrm{~kg}$ were quite similar to the overall dissolution rate (45.1\%) and response rate (47.2\%). Another factor that should be taken into account during gallstone dissolution is the cost of the bile acid, albeit not directly related to successful response to bile acid therapy. As mentioned above, three capsules of $\mathrm{CNU}^{\circledR}$ per day is the dose that is covered by the National Health Insurance Program of Korea. Therefore, the total cost of $\mathrm{CNU}^{\circledR}$ itself that a patient has to pay for 6 months is about 143 United States Dollar (USD). The corresponding cost of UDCA $600 \mathrm{mg} /$ day is about 89 USD and that of UDCA 900 $\mathrm{mg} /$ day is about 134 USD. Therefore, different from the Western countries, combination of UDCA and CDCA is not a cheaper alternative. However, since the cost of medication comprises about $12 \%$ to $17 \%$ of the total medical cost related to gallstone dissolution, the cost-effectiveness of $\mathrm{CNU}^{\circledR}$ and UDCA could be said to be comparable.

As for gallstone-related symptoms, symptom improvement was observed independent of gallstone dissolution with most of the symptoms showing greatest improvement during the first 2 weeks. Both the number of patients with symptoms (Fig. 3A and $\mathrm{B}$ ) and the symptom score (Fig. 3C and D) decreased with $\mathrm{CNU}^{\circledR}$ administration. Previous reports have shown that dyspepsia and biliary pain improved with either CDCA or UDCA. ${ }^{27,28}$ Combination therapy with CDCA and UDCA has also shown a decrease in the percentage of patients experiencing biliary pain. ${ }^{5}$ Although the aforementioned studies did not compare symptom improvement according to response to therapy, our study demonstrated that there was symptom improvement regardless of gallstone dissolution. This may result from the dissolution of cholesterol crystals and anti-inflammatory effect of bile acid, especially by hydrophilic UDCA. ${ }^{29}$

Different from previous studies which employed oral cholecystography to measure and calculate stone volume, ${ }^{11}$ we used ultrasonography to measure the gallstone diameter. Ultrasonography is now considered the best method for diagnosing gallstone disease due to its noninvasiveness, safety, wide availability, and high sensitivity and specificity for detecting GB stones. ${ }^{2,30}$ Since oral cholecystography is an obsolete study, it would have been impractical to evaluate the dissolution rate with oral cholecystography. Thus, using ultrasonography is better for reflecting the contemporary clinical practice. Another difference from previous studies is that the present study used diisopropyl iminodiacetic acid scan to measure GBEF. Previous studies on dissolution efficacy used either oral cholecystography or ultrasonography to select 'functioning' GB..$^{5,10,11,30,31}$ Although the normal value of GBEF is not clearly defined, it is accepted to be over $75 \%$ with cutoff value always being $>35 \%$. $^{32}$ The cutoff value of GBEF in the current study was $>50 \%$. Therefore, those with truly functioning GB were selected. Since the degree of "function" could better be delineated, we expected to see some relationship between GBEF and dissolution rate. However, our results point out that as long as GBEF is above 50\%, GBEF does not affect stone dissolution.

In terms of adverse events, diarrhea that led to discontinuation of dissolution therapy with $\mathrm{CNU}^{\circledR}$ occurred only in one subject. In addition, hepatotoxicity did not occur during the study period. Although three subjects experienced acute pancreatitis $(n=1)$, acute cholecystitis $(n=1)$, and severe abdominal pain $(n=1)$, these adverse events may not be related to dissolution therapy with $\mathrm{CNU}^{\circledR}$ but could be the natural course of gallstone itself. The drop-out rate of enrolled subjects in the present study, including those who experienced adverse events, was $17.7 \%$ (42/237). This rate is similar to previous studies; Podda et al., ${ }^{10}$ Jazrawi et al., ${ }^{11}$ and Petroni et al. ${ }^{5}$ reported drop-out rates of 12.5\% (15/120), 17.2\% (10/58), and 39.6\% (61/154), each. 
However, our study was carried out for 6 months whereas all the previous studies have been carried out for 2 year. Therefore, the drop-out rate in our study could become higher if the study period had been longer.

There are few limitations to this study. First, our study was conducted only for 6 months, so the long-term efficacy of $\mathrm{CNU}^{\circledR}$ and recurrence of gallstones in patients with response to bile acid therapy could not be assessed. According to previous studies, complete dissolution rate increased up to 24 months with combination therapy of CDCA and UDCA., ${ }^{5,10}$ If our study period had been longer, the dissolution rate and percentage of patients with complete dissolution could have increased. Secondly, patients with high BMI $\left(\geq 35 \mathrm{~kg} / \mathrm{m}^{2}\right)$ were excluded. Patients with elevated BMI are at a greater risk of developing cholesterol gallstones and becoming symptomatic. ${ }^{33}$ Therefore, it would be reasonable to include these patients in the future studies since they are the ones who would benefit the most from dissolution therapy.

In conclusion, $\mathrm{CNU}^{\circledR}$ was well-tolerated and effective for dissolving gallstones in about half of patients after therapy with $\mathrm{CNU}^{\circledR}$ for 6 months. Treatment for uncomplicated asymptomatic gallstone is currently not recommended due to low incidence rate of complications. However, considering the long life expectancy and increased gallstone incidence among young people, there is a strong likelihood of developing complication during lifetime. Therefore, more aggressive therapeutic approach for gallstone should be considered.

\section{CONFLICTS OF INTEREST}

No potential conflict of interest relevant to this article was reported.

\section{ACKNOWLEDGEMENTS}

This research was funded by Myungmoon Pharmaceutical Company which provided the cost for the following examinations after enrollment: DISIDA scan, follow-up abdominal sonography, and follow-up laboratory studies.

\section{REFERENCES}

1. Chang YR, Jang JY, Kwon W, et al. Changes in demographic features of gallstone disease: 30 years of surgically treated patients. Gut Liver 2013;7:719-724.

2. Portincasa P, Moschetta A, Palasciano G. Cholesterol gallstone disease. Lancet 2006;368:230-239.

3. Jeong SU, Lee SK. Obesity and gallbladder diseases. Korean J Gastroenterol 2012;59:27-34.

4. Kim JW, Oh HC, Do JH, Choi YS, Lee SE. Has the prevalence of cholesterol gallstones increased in Korea? A preliminary singlecenter experience. J Dig Dis 2013;14:559-563.
5. Petroni ML, Jazrawi RP, Pazzi P, et al. Ursodeoxycholic acid alone or with chenodeoxycholic acid for dissolution of cholesterol gallstones: a randomized multicentre trial. The British-Italian Gallstone Study group. Aliment Pharmacol Ther 2001;15:123-128.

6. Sleisenger MH, Fordtran JS, Feldman ML, Friedman LS, Brandt LJ. Sleisenger and Fordtran's gastrointestinal and liver disease: pathophysiology, diagnosis, management. Volume 2. 9th ed. Philadelphia: Saunders/Elsevier, 2010:1122.

7. May GR, Sutherland LR, Shaffer EA. Efficacy of bile acid therapy for gallstone dissolution: a meta-analysis of randomized trials. Aliment Pharmacol Ther 1993;7:139-148.

8. Raedsch R, Stiehl A, Czygan P. Ursodeoxycholic acid and gallstone calcification. Lancet 1981;2:1296.

9. Bateson MC, Bouchier IA, Trash DB, Maudgal DP, Northfield TC. Calcification of radiolucent gall stone during treatment with ursodeoxycholic acid. Br Med J (Clin Res Ed) 1981;283:645-646.

10. Podda M, Zuin M, Battezzati PM, Ghezzi C, de Fazio C, Dioguardi ML. Efficacy and safety of a combination of chenodeoxycholic acid and ursodeoxycholic acid for gallstone dissolution: a comparison with ursodeoxycholic acid alone. Gastroenterology 1989;96: 222-229.

11. Jazrawi RP, Pigozzi MG, Galatola G, Lanzini A, Northfield TC. Optimum bile acid treatment for rapid gall stone dissolution. Gut 1992;33:381-386.

12. Danzinger RG, Hofmann AF, Schoenfield LJ, Thistle JL. Dissolution of cholesterol gallstones by chenodeoxycholic acid. N Engl J Med 1972;286:1-8.

13. Bell GD, Whitney B, Dowling RH. Gallstone dissolution in man using chenodeoxycholic acid. Lancet 1972;2:1213-1216.

14. Nakagawa S, Makino I, Ishizaki T, Dohi I. Dissolution of cholesterol gallstones by ursodeoxycholic acid. Lancet 1977;2:367-369.

15. Tint GS, Salen G, Colalillo A, et al. Ursodeoxycholic acid: a safe and effective agent for dissolving cholesterol gallstones. Ann Intern Med 1982;97:351-356.

16. Diehl AK. Epidemiology and natural history of gallstone disease. Gastroenterol Clin North Am 1991;20:1-19.

17. Kim MH, Lim BC, Myung SJ, et al. Epidemiological study on Korean gallstone disease: a nationwide cooperative study. Dig Dis Sci 1999;44:1674-1683.

18. Trotman BW, Petrella EJ, Soloway RD, Sanchez HM, Morris TA 3rd, Miller WT. Evaluation of radiographic lucency or opaqueness of gallstones as a means of identifying cholesterol or pigment stones: correlation of lucency or opaqueness with calcium and mineral. Gastroenterology 1975;68:1563-1566.

19. Bell GD, Dowling RH, Whitney B, Sutor DJ. The value of radiology in predicting gallstone type when selecting patients for medical treatment. Gut 1975;16:359-364.

20. Baron RL, Rohrmann CA Jr, Lee SP, Shuman WP, Teefey SA. CT evaluation of gallstones in vitro: correlation with chemical analysis. AJR Am J Roentgenol 1988;151:1123-1128.

21. Janowitz P, Zöller A, Swobodnik W, Wechsler JG, Schumacher KA, Ditschuneit H. Computed tomography evaluation of radiolu- 
cent gallstones in vivo. Gastrointest Radiol 1990;15:58-60.

22. Whiting MJ, Jarvinen V, Watts JM. Chemical composition of gallstones resistant to dissolution therapy with chenodeoxycholic acid. Gut 1980;21:1077-1081.

23. Freilich HS, Malet PF, Schwartz JS, Soloway RD. Chemical and morphologic characteristics of cholesterol gallstones that failed to dissolve on chenodiol: the National Cooperative Gallstone Study. Gastroenterology 1986;91:713-718.

24. Kupfer RM, Maudgal DP, Northfield TC. Gallstone dissolution rate during chenic acid therapy: effect of bedtime administration plus low cholesterol diet. Dig Dis Sci 1982;27:1025-1029.

25. Lanzini A, Facchinetti D, Northfield TC. Maintenance of hepatic bile acid secretion rate during overnight fasting by bedtime bile acid administration. Gastroenterology 1988;95:1029-1035.

26. Inoi J, Shimizu I, Tsuji Y, Muguruma N, Shibata H, Ito S. Effect of administration of ursodeoxycholic acid at bedtime on cholesterol saturation of hepatic bile in Japanese patients with gallstone. J Med Invest 1998;45:115-122.

27. Polli EE, Bianchi PA, Conte D, Sironi L. Treatment of radiolucent gallstones with CDCA or UDCA: a multicenter trial. Digestion 1981;22:185-191.
28. Meredith TJ, Williams GV, Maton PN, Murphy GM, Saxton HM, Dowling RH. Retrospective comparison of 'Cheno' and 'Urso' in the medical treatment of gallstones. Gut 1982;23:382-389.

29. Guarino MP, Cong P, Cicala M, Alloni R, Carotti S, Behar J. Ursodeoxycholic acid improves muscle contractility and inflammation in symptomatic gallbladders with cholesterol gallstones. Gut 2007; 56:815-820.

30. Portincasa P, Ciaula AD, Bonfrate L, Wang DQ. Therapy of gallstone disease: what it was, what it is, what it will be. World J Gastrointest Pharmacol Ther 2012;3:7-20.

31. Tuncer I, Harman M, Colak Y, Arslan I, Turkdogan MK. Effect of ursodeoxycholic acid alone and ursodeoxycholic acid plus domperidone on radiolucent gallstones and gallbladder contractility in humans. Gastroenterol Res Pract 2012;2012:159438.

32. DiBaise JK, Oleynikov D. Does gallbladder ejection fraction predict outcome after cholecystectomy for suspected chronic acalculous gallbladder dysfunction? A systematic review. Am J Gastroenterol 2003;98:2605-2611.

33. Stender S, Nordestgaard BG, Tybjaerg-Hansen A. Elevated body mass index as a causal risk factor for symptomatic gallstone disease: a Mendelian randomization study. Hepatology 2013;58:2133-2141. 